\title{
Proper complex random processes
}

\author{
Yu. V. Kozachenko ${ }^{1}$, M.Yu. Petranova ${ }^{2, *}$ \\ ${ }^{1}$ Department of Probability Theory, Statistics and Actuarial Mathematics, Taras Shevchenko National University of Kyiv, Ukraine, \\ Department of Probability Theory and Mathematical Statistics, Vasyl’ Stus Donetsk National University, Ukraine, yvk@univ.kiev.ua. \\ ${ }^{2}$ Department of Probability Theory and Mathematical Statistics, Vasyl' Stus Donetsk National University, Ukraine, \\ m.petranova@donnu.edu.ua.
}

(Received: 12 February 2017; Accepted: 13 May 2017)

\begin{abstract}
In this paper we study properties of stationary proper complex random process with stable correlation functions. Estimates are obtained for distribution of supremum of modulus of these processes and normes in spaces $L_{p}$ on finite and infinite intervals.
\end{abstract}

Keywords complex random process, stationary Gaussian proper complex random process, stable correlation function, square Gaussian random process.

\section{AMS 2010 subject classifications 60G15.}

DOI: $10.19139 /$ soic.v5i2.276

\section{Introduction}

This article deals with complex random processes which are one of the most important generalizations of the concept of random process (see $[2,12])$. The complex random processes are especially relevant when the narrowbanded processes are investigated. These processes are exploited as models of complex amplitudes of quasiharmonic oscillations or waves in radiophysics and optics [1]. In this article we presented results of investigation of properties of complex random processes which are useful when solving problems in the listed above areas. Conditions for existence of proper complex random processes are described in [12, 2]. In this article we investigate stationary proper complex random processes, stationary proper complex random processes with stable correlation function. Some results for properties of stable correlation function are presented in paper[11]. In this article some properties of square Gaussian random variables and process are presented (for more results see, for example, $[3,7,8])$. Also, in this paper estimates of distributions of functionals from the module of stationary Gaussian proper complex random processes are obtained (for more results see, for example, $[13,6,10])$. Theorems, which describe behavior of the module of stationary proper complex random process at infinity are developed.

The content of the article is as follows. In Section 2 we introduce the basic definitions related to the complex random processes. Stationary proper complex random processes are introduced and discussed in Section 3. In the next Section 4, we deal with properties of square Gaussian random variables and processes. Section 5 is related to estimates of distributions of some functions from the module of stationary Gaussian proper complex random process. And in the last Section 6 behavior of the module of stationary proper complex random process at infinity is studied.

\footnotetext{
*Correspondence to: 21, 600-richya str., Vinnytsia, Department of Probability Theory and Mathematical Statistics, Vasyl' Stus Donetsk National University, Ukraine (m.petranova@ donnu.edu.ua).
}

ISSN 2310-5070 (online) ISSN 2311-004X (print)

Copyright (C) 2017 International Academic Press 


\section{Proper Complex Random Process}

Definition 2.1. A random process of the form $X(t)=X_{c}(t)+i X_{s}(t), t \in \mathbb{R}$, where $X_{c}(t)$ and $X_{s}(t)$ are realvalued random processes ( $c$ - cosine, $s-$ sine), is called complex random process (see book [2] and paper [12]).

Remark 2.1. In this paper we will consider centered random processes, that is

$$
E X(t)=E X_{c}(t)=E X_{s}(t)=0 .
$$

Definition 2.2. The function

$$
\begin{gathered}
r(\tau, t)=E X(t+\tau) \bar{X}(t)=E X_{c}(t+\tau) X_{c}(t)+E X_{s}(t+\tau) X_{s}(t)+ \\
+i\left(E X_{s}(t+\tau) X_{c}(t)-E X_{c}(t+\tau) X_{s}(t)\right)
\end{gathered}
$$

is called correlation function of the process $X(t)$.

The function

$$
\begin{gathered}
\widehat{r}(\tau, t)=E X(t+\tau) X(t)= \\
=E X_{c}(t+\tau) X_{c}(t)-E X_{s}(t+\tau) X_{s}(t)+i\left(E X_{c}(t+\tau) X_{s}(t)+E X_{s}(t+\tau) X_{c}(t)\right)
\end{gathered}
$$

is called pseudo correlation function of the process $X(t)$.

Definition 2.3. A complex random process $X(t)$ is called proper complex random process (PCR process), if the pseudo correlation function of this process is equal to zero, $E X(t+\tau) X(t)=0$, that is when conditions

$$
\begin{gathered}
E X_{c}(t+\tau) X_{c}(t)=E X_{s}(t+\tau) X_{s}(t), \\
E X_{c}(t+\tau) X_{s}(t)=-E X_{s}(t+\tau) X_{c}(t) .
\end{gathered}
$$

hold true.

Remark 2.2. Conditions under which PCR processes exist are described in book [2] and paper [12].

Definition 2.4. [12] A proper complex random process is called (wide sense) stationary if for all $\tau, t \in \mathbb{R}$ the following relation holds true

$$
r(\tau, t)=E X(t+\tau) \bar{X}(t)=r(\tau) .
$$

Remark 2.3. In the case where PCR-process $X(t)$ is stationary we can write the following relations

$$
\begin{gathered}
E X_{c}(t+\tau) X_{c}(t)=E X_{s}(t+\tau) X_{s}(t)=\frac{1}{2} \operatorname{Re} r(\tau), \\
E X_{c}(t+\tau) X_{s}(t)=\frac{1}{2} \operatorname{Im} r(\tau) .
\end{gathered}
$$

Definition 2.5. A complex random process $X(t)=X_{c}(t)+i X_{s}(t)$ is called Gaussian if the real-valued random processes $X_{c}(t)$ and $X_{s}(t)$ are jointly Gaussian processes.

\section{Stationary PCR-processes with stable correlation functions}

Definition 3.1. The correlation function $r(\tau), \tau \in \mathbb{R}$ of stationary proper complex random process is called stable correlation function if it can be represented in the form

$$
r(\tau)=\sigma^{2} \exp \left\{-c|\tau|^{\alpha}\left(1+i \beta \frac{\tau}{|\tau|} \omega(\tau, \alpha)\right)\right\}
$$

where $\sigma^{2}, c, \beta, \alpha$ are real-valued constants, such that $\sigma^{2}>0, c>0,|\beta| \leq 1,0 \leq \alpha \leq 2$,

$$
\omega(\tau, \alpha)=\left\{\begin{array}{l}
\operatorname{tg} \frac{\pi \alpha}{2}, 0 \leq \alpha \leq 2, \alpha \neq 1, \\
\frac{2}{\pi} \log |\tau|, \alpha=1 .
\end{array}\right.
$$


Remark 3.1. The function $r(\tau)$ is non-negative definite, since $r(\tau)$ is the characteristic function of a stable random variable $\xi, E \xi=0$, in the case where $\sigma^{2}=1$ (see [11, p.169]).

Definition 3.2. A stationary PCR process is called proper stationary random complex process with stable covariance function (stationary SPCR process) if

$$
E X(t+\tau) \bar{X}(t)=r(\tau)
$$

where the function $r(\tau)$ is given by formula (5).

Remark 3.2. For the proper stationary random complex process $X(t)=X_{c}(t)+i X_{s}(t), E X(t)=0$, with stable covariance functions the following relations hold true

$$
\begin{gathered}
E X_{c}(t+\tau) X_{c}(t)=\frac{1}{2} \operatorname{Re} r(\tau)= \\
=\frac{\sigma^{2}}{2} \exp \left\{-c|\tau|^{\alpha}\right\} \cos \left(c|\tau|^{\alpha} \beta \frac{\tau}{|\tau|} \omega(\tau, \alpha)\right)= \\
=E X_{s}(t+\tau) X_{s}(t), \\
E X_{c}(t+\tau) X_{s}(t)=\frac{1}{2} \operatorname{Im} r(\tau)= \\
=-\frac{\sigma^{2}}{2} \exp \left\{-c|\tau|^{\alpha}\right\} \sin \left(c|\tau|^{\alpha} \beta \frac{\tau}{|\tau|} \omega(\tau, \alpha)\right), \\
\operatorname{Re} r(-\tau)=\operatorname{Re} r(\tau) .
\end{gathered}
$$

\section{Square Gaussian random variables and processes}

In this section we propose definitions and some properties of square Gaussian random variables and processes.

Definition 4.1. [3, 7] Let $(T, \rho)$ be a metric space and let $\Theta=\{\xi(t), t \in T\}, E \xi(t)=0$, be a family of jointly Gaussian random variables (e.g. $\xi=\{\xi(t), t \in T\}$ is a Gaussian random process). The space of square Gaussian random variable $\left(S G_{\Theta}(\Omega)\right)$ is such a space that any element $\eta \in S G_{\Theta}(\Omega)$ can be presented in the form

$$
\eta=\vec{\xi}^{\top} A \vec{\xi}-E\left(\vec{\xi}^{\top} A \vec{\xi}\right),
$$

where $\vec{\xi}^{\top}=\left(\xi_{1}, \xi_{2}, \ldots, \xi_{n}\right), \xi_{k} \in \Theta, k=1,2, \ldots, n$ and $A$ is a real-valued matrix, or the element $\eta \in S G_{\Theta}(\Omega)$ is the mean square limit of a sequence of random variables of the form (9):

$$
\eta=\operatorname{l.i.m} .\left(\vec{\xi}_{n}^{\top} A_{n} \vec{\xi}_{n}-E\left(\vec{\xi}_{n}^{\top} A \vec{\xi}_{n}\right)\right) .
$$

Definition 4.2. A random process $\eta=\{\eta(t), t \in T\}$ is called square Gaussian process if the family of random variables $\eta=\{\eta(t), t \in T\}$ forms the space of square Gaussian random variables.

The next theorem is a modification of Theorem 3.2 from the book [7].

\section{Theorem 4.1}

Let $X=\{X(t), t \in[a, b]\}$ be a separable square Gaussian random process and let the condition

$$
\sup _{|t-s| \leq h}(\operatorname{Var}(X(t)-X(s)))^{1 / 2} \leq C h^{\beta}
$$

holds true for $\beta \in(0,1], C>0$. Then for all integer $M>1$ and all

$$
x>\frac{\sqrt{2} \gamma_{0} M}{\beta} \max \left(1,\left(\frac{b-a}{2}\right)^{\beta} \frac{C}{\gamma_{0}}\right)^{\frac{1}{M-1}},
$$


where $\gamma_{0}=\sup _{a \leq t \leq b}(\operatorname{Var} X(t))^{1 / 2}$, the tail of distribution of the process $|X(t)|$ can be estimated in the following way

$$
P\left\{\sup _{t \in[a, b]}|X(t)|>x\right\} \leq 4 e^{\frac{M+1}{\beta}} \cdot \exp \left\{-\frac{x}{\sqrt{2} \gamma_{0}}\right\}\left(\frac{\beta \cdot x}{\sqrt{2} \gamma_{0} M}\right)^{\frac{M}{\beta}}\left(1+\frac{\sqrt{2} x}{\gamma_{0}}\right)^{1 / 2} .
$$

Theorem 4.2

[9] Let $X=\{X(t), t \in[a, b]\}$, where $-\infty \leq a<b \leq \infty$, be a measurable square-Gaussian random process. Let the Lebesgue integral

$$
\int_{a}^{b}\left(E(X(t))^{2}\right)^{p / 2} d t
$$

be well defined for $p \geq 1$. Then the integral

$$
\int_{a}^{b}|X(t)|^{p} d t
$$

exists with probability 1 and for all

$$
\varepsilon \geq\left(\frac{p}{\sqrt{2}}+\sqrt{\left(\frac{p}{2}+1\right) p}\right)^{p} C_{p}
$$

where

$$
C_{p}=\int_{a}^{b}\left(E(X(t))^{2}\right)^{p / 2} d t
$$

the following inequality holds true

$$
P\left\{\int_{a}^{b}|X(t)|^{p} d t>\varepsilon\right\} \leq 2 \sqrt{1+\frac{\varepsilon^{1 / p} \sqrt{2}}{C_{p}^{1 / p}}} \exp \left\{-\frac{\varepsilon^{1 / p}}{\sqrt{2} C_{p}^{1 / p}}\right\} .
$$

Corollary 4.1

Let assumptions of Theorem 4.2 be satisfied. Then for

$$
u \geq\left(\frac{p}{\sqrt{2}}+\sqrt{\left(\frac{p}{2}+1\right) p}\right) C_{p}^{1 / p}
$$

the following inequality holds true

$$
P\left\{\|X(t)\|_{L_{p}(a, b)}>u\right\} \leq 2 \sqrt{1+\frac{u \sqrt{2}}{C_{p}^{1 / p}}} \cdot \exp \left\{-\frac{u}{\sqrt{2} C_{p}^{1 / p}}\right\} .
$$

\section{Estimation of distribution of some functionals from module of stationary Gaussian PCR-processes}

Theorem 5.1

Let $X=\{X(t), t \in[a, b]\}$ be a Gaussian SPCR process and let $|X(t)|=\left(X_{c}^{2}(t)+X_{s}^{2}(t)\right)^{1 / 2}$. Then for

$$
u \geq\left(\frac{p}{\sqrt{2}}+\sqrt{\left(\frac{p}{2}+1\right) p}\right) \sigma^{2}(b-a)^{1 / p}
$$


the following inequality holds

$$
P\left\{\left\|X(t)^{2}-\sigma^{2}\right\|_{L_{p}([a, b])}>u\right\} \leq 2 \sqrt{1+\frac{u \sqrt{2}}{(b-a)^{1 / p} \sigma^{2}}} \cdot \exp \left\{-\frac{u}{\sqrt{2}(b-a)^{1 / p} \sigma^{2}}\right\} .
$$

Proof

The proof of this theorem follows from inequality (13). Indeed it follows from (6), that

$$
E X_{c}^{2}(t)=E\left(X_{s}(t)\right)^{2}=\frac{1}{2} R(r(0))=\frac{\sigma^{2}}{2} .
$$

Therefore $E|X(t)|^{2}=\sigma^{2}$ and

$$
E\left(|X(t)|^{2}-\sigma^{2}\right)^{2}=E\left(|X(t)|^{2}-E|X(t)|^{2}\right)^{2}=E|X(t)|^{4}-\left(E|X(t)|^{2}\right)^{2}=E|X(t)|^{4}-\sigma^{4} .
$$

Suppose that $\left(X_{1}, X_{2}, X_{3}, X_{4}\right)$ is a zero-mean Gaussian vector. Then we have:

$$
E\left(X_{1} X_{2} X_{3} X_{4}\right)=E\left(X_{1} X_{2}\right) E\left(X_{3} X_{4}\right)+E\left(X_{1} X_{3}\right) E\left(X_{2} X_{4}\right)+E\left(X_{1} X_{4}\right) E\left(X_{2} X_{3}\right) .
$$

This equality is called Isserlis formula (see, for example [3, p.228]. Making use of this formula and relations (3), (4) we can write

$$
\begin{gathered}
E|X(t)|^{4}=E\left(\left|X_{c}(t)\right|^{2}+\left|X_{s}(t)\right|^{2}\right)^{2}=E\left|X_{c}(t)\right|^{4}+E\left|X_{s}(t)\right|^{4}+2 E\left|X_{c}(t)\right|^{2}\left|X_{s}(t)\right|^{2}, \\
E\left|X_{c}(t)\right|^{4}=3\left(E\left|X_{c}(t)\right|^{2}\right)^{2}=3 \frac{\sigma^{4}}{4}=E\left|X_{s}(t)\right|^{4} \\
E\left(\left|X_{c}(t)\right|^{2}\left|X_{s}(t)\right|^{2}\right)=E\left|X_{c}(t)\right|^{2} E\left|X_{s}(t)\right|^{2}+2\left(E X_{c}(t) X_{s}(t)\right)^{2} .
\end{gathered}
$$

Since

we have

$$
E\left(X_{c}(t) X_{s}(t)\right)=\frac{1}{2} \operatorname{Im}(r(0))=0
$$

It follows from (15) that

$$
E|X(t)|^{4}=2 \sigma^{4}
$$

$$
E\left((X(t))^{2}-\sigma^{2}\right)^{2}=\sigma^{4}
$$

and

$$
\int_{a}^{b} E\left(|X(t)|^{2}-\sigma^{2}\right)^{\frac{p}{2}} d t=\sigma^{2 p}(b-a)
$$

Now (14) follows from (13).

Theorem 5.2

Let $X=\{X(t), t \in[a, b]\}$ be a Gaussian SPCR process and let

$$
|X(t)|=\left(X_{c}^{2}(t)+X_{s}^{2}(t)\right)^{1 / 2} .
$$

If $X(t)$ is a separable process, then for all integer $M>1$ and all

$$
u>\frac{2 \sqrt{2} \sigma^{2} M}{\alpha}\left(\max \left(1,\left(\frac{b-a}{2}\right)^{\alpha / 2} 2 \sqrt{c}\right)^{\frac{1}{M-1}}\right)
$$


we have

$$
P\left\{\sup _{a \leq t \leq b}\left|(X(t))^{2}-\sigma^{2}\right|>x\right\} \leq 4 e^{\frac{2(M+1)}{\alpha}} \exp \left\{-\frac{x}{\sqrt{2} \sigma^{2}}\right\}\left(\frac{\alpha x}{2 \sqrt{2} \sigma^{2} M}\right)^{\frac{2 M}{\alpha}}\left(1+\frac{\sqrt{2} x}{\sigma^{2}}\right)^{1 / 2}
$$

\section{Proof}

The statement of this Theorem follows from Theorem 4.1. In our case $\gamma_{0}=\sigma^{2}$.

In order to to apply Theorem 4.1 to the process $|X(t)|^{2}=\left(X_{c}^{2}(t)+X_{s}^{2}(t)\right)^{2}$ we have to astimate $E(Y(t)-Y(s))^{2}$, where $Y(t)=|X(t)|^{2}-\sigma^{2}$.

It is easy to see that

$$
\begin{gathered}
E(Y(t)-Y(s))^{2}=E\left(X_{c}^{2}(t)+X_{s}^{2}(t)-X_{c}^{2}(s)-X_{s}^{2}(s)\right)^{2}= \\
=E\left(X_{c}^{2}(t)-X_{c}^{2}(s)+X_{s}^{2}(t)-X_{s}^{2}(s)\right)^{2}= \\
=E\left(X_{c}^{2}(t)-X_{c}^{2}(s)\right)^{2}+E\left(X_{s}^{2}(t)-X_{s}^{2}(s)\right)^{2}+2 E\left(X_{c}^{2}(t)-X_{c}^{2}(s)\right) E\left(X_{s}^{2}(t)-X_{s}^{2}(s)\right)= \\
w_{1}+w_{2}+w_{3}, \\
w_{1}=E\left(X_{c}(t)\right)^{4}+E\left(X_{c}(s)\right)^{4}-2 E\left(X_{c}^{2}(t) X_{c}^{2}(s)\right), \\
E\left(X_{c}(t)\right)^{4}=E\left(X_{c}(s)\right)^{4}=\frac{3}{4} \sigma^{4}, \\
E\left(X_{c}^{2}(t) X_{c}^{2}(s)\right)=E\left(X_{c}(t)\right)^{2} E\left(X_{c}(s)\right)^{2}+2\left(E X_{c}(t) X_{c}(s)\right)^{2}= \\
=\frac{\sigma^{4}}{4}+2\left(\frac{1}{2} \operatorname{Re}(r(t-s))^{2}\right.
\end{gathered}
$$

Therefore

$$
w_{1}=\sigma^{4}-(\operatorname{Re}(r(t-s)))^{2}
$$

Next; we have

$$
\begin{gathered}
w_{1}=w_{2} \\
w_{1}+w_{2}=2\left(\sigma^{4}-(\operatorname{Re}(r(t-s)))^{2}\right), \\
\frac{w_{3}}{2}=E\left(X_{c}^{2}(t) X_{s}^{2}(t)\right)+E\left(X_{c}^{2}(s) X_{s}^{2}(s)\right)-E\left(X_{c}^{2}(s) X_{s}^{2}(t)\right)-E\left(X_{c}^{2}(t) X_{s}^{2}(s)\right) .
\end{gathered}
$$

Since $\operatorname{Im}(r(0))=0$, then

$$
E\left(X_{c}^{2}(t) X_{s}^{2}(t)\right)=E X_{c}^{2}(t) E X_{s}^{2}(t)+2\left(E X_{c}(t) X_{s}(t)\right)^{2}=\frac{\sigma^{4}}{4}+2\left(\frac{1}{2} \operatorname{Im}(r(0))\right)^{2}=\frac{\sigma^{4}}{4} .
$$

In the same way we can obtaine that

$$
\begin{gathered}
E\left(X_{c}^{2}(s) X_{s}^{2}(s)\right)=\frac{\sigma^{4}}{4} \\
E\left(X_{c}^{2}(t) X_{s}^{2}(s)\right)=\frac{\sigma^{4}}{4}+\frac{1}{2}(\operatorname{Im}(r(t-s)))^{2} .
\end{gathered}
$$

Consequently

$$
w_{3}=-\left((\operatorname{Im}(r(s-t)))^{2}+(\operatorname{Im}(r(t-s)))^{2}\right) .
$$

Since

$$
(\operatorname{Im}(r(s-t)))^{2}=(\operatorname{Im}(r(t-s)))^{2}
$$

we have

$$
w_{3}=-2(\operatorname{Im}(r(t-s)))^{2}
$$


and

$$
E(Y(t)-Y(s))^{2}=2\left(\sigma^{4}-\left((\operatorname{Re}(r(t-s)))^{2}+(\operatorname{Im}(r(t-s)))^{2}\right)\right) .
$$

Since

$$
\begin{gathered}
(\operatorname{Re}(r(t-s)))^{2}+(\operatorname{Im}(r(t-s)))^{2}=|r(t-s)|^{2}=\sigma^{4} \exp \{-2 c(t-s)\} \\
=\left|\cos \left(-c\left|\tau^{\alpha}\right| i \beta \frac{(t-s)}{|t-s|} w(t, \alpha)\right)+i \sin \left(-c\left|\tau^{\alpha}\right| i \beta \frac{(t-s)}{|t-s|} w(t, \alpha)\right)\right|^{2}= \\
=\sigma^{2} \exp \left\{-|t-s|^{\alpha} c\right\}
\end{gathered}
$$

we get the following estimate

$$
E(Y(t)-Y(s))^{2}=2 \sigma^{4}\left(1-\exp \left\{-2 c|t|^{\alpha}\right\}\right) \leq 4 \sigma^{4} c|t|^{\alpha} .
$$

Consequently $\beta=\frac{\alpha}{2}$ and $C=2 \sigma^{2} \sqrt{c}$. Therefore (16) follows from (11).

\section{Behavior of the module of stationary PCR-process at infinity}

Theorem 6.1

Let $X=\{X(t), t \in(-\infty, \infty)\}$ be a measurable Gaussian SPCR process, let $|X(t)|=\left(X_{c}^{2}(t)+X_{s}^{2}(t)\right)^{1 / 2}$ and let $Y(t)=|X(t)|^{2}-E(X(t))^{2}=|X(t)|^{2}-\sigma^{2}$. Let $c(t), t \in R$ be a function such that

$$
\int_{-\infty}^{\infty}|c(t)|^{-p} d t<\infty, p \geq 1
$$

Then for

$$
u \geq\left(\frac{p}{\sqrt{2}}+\sqrt{\left(\frac{p}{2}+1\right) p}\right) \cdot \sigma^{2}\left(\int_{-\infty}^{\infty}|c(t)|^{-p} d t\right)^{1 / p}
$$

the following inequality holds true

$$
P\left\{\left\|\frac{(X(t))^{2}-\sigma^{2}}{c(t)}\right\|_{L_{p}(-\infty, \infty)}>u\right\} \leq 2 \sqrt{1+\frac{\sqrt{2} u}{\left(\int_{-\infty}^{\infty}|c(t)|^{-p} d t\right)^{1 / p} \sigma^{2}}} \cdot \exp \left\{-\frac{u}{\sqrt{2}\left(\int_{-\infty}^{\infty}|c(t)|^{-p} d t\right)^{1 / p}}\right\} .
$$

Proof

The statement of this Theorem follows from Theorem 4.2 (see Corollary 4.1) if we take

$$
C_{p}=2^{-\frac{p}{2}} \sigma^{2 p} \cdot \int_{-\infty}^{\infty}|c(t)|^{-p} d t
$$

\section{Corollary 6.1}

Let $c(t)>0$ be an even monotone increasing function for which conditions of Theorem 6.1 are satisfied. Then for all $t \geq 0$ the following inequality holds true with probability one:

$$
\left(\int_{-t}^{t}\left|(X(u))^{2}-\sigma^{2}\right|^{p} d u\right)^{1 / p} \leq c(t) \cdot \xi
$$


where $\xi>0$ is a random variable such that

$$
P\{\xi>u\} \leq 2 \sqrt{1+\frac{\sqrt{2} u}{\left(\int_{-\infty}^{\infty}|c(t)|^{-p} d t\right)^{1 / p} \cdot \sigma^{2}}} \cdot \exp \left\{-\frac{u}{\left(\sqrt{2} \int_{-\infty}^{\infty}|c(t)|^{-p} d t\right)^{1 / p} \cdot \sigma^{2}}\right\},
$$

for

$$
u \geq\left(\frac{p}{\sqrt{2}}+\sqrt{\left(\frac{p}{2}+1\right) p}\right) \cdot \sigma^{2}\left(\int_{-\infty}^{\infty}|c(t)|^{-p} d t\right)^{1 / p}
$$

Proof

The statement of Corollary 6.1 follows from inequalities: $t \geq 0$,

$$
\begin{gathered}
\left(\int_{-t}^{t}\left|(X(u))^{2}-\sigma^{2}\right|^{p} d u\right)^{1 / p} \leq c(t) \cdot\left(\int_{-t}^{t} \frac{\left|(X(u))^{2}-\sigma^{2}\right|^{p}}{c(u)^{p}} d u\right)^{1 / p} \leq \\
\leq c(t) \cdot\left\|\frac{\left|(X(t))^{2}-\sigma^{2}\right|}{c(t)}\right\|_{L_{p}(-\infty, \infty)} \cdot
\end{gathered}
$$

Remark 6.1. The statement of Corollary 6.1 holds true, for example, for function $c(t)=\left(1+|t|^{\gamma}\right), \gamma>p$.

Theorem 6.2

Let $X=\{X(t), t \in[a, b]\}$ be a separable Gaussian stationary PCR process. Let there exist a sequence $a_{k}, k=$ $0,1,2, \ldots$ such that $a_{k}<a_{k+1}, a_{k} \rightarrow \infty$, as $k \rightarrow \infty, a_{0}=0$ and a function $c(t), t \in[0, \infty)$ such that $c(t) \geq 1, c(t)$ be an even monotone increasing continuous and $c(t) \rightarrow \infty$ if $t \rightarrow \infty, Y(t)=|X(t)|^{2}-E(X(t))^{2}=|X(t)|^{2}-\sigma^{2}$. Let the condition

$$
\varepsilon^{*}=\frac{2 \sqrt{2} \sigma^{2} M}{\alpha} \sup _{0 \leq k \leq \infty} \frac{1}{c\left(a_{k}\right)} \max \left(1,\left(\left(\frac{a_{k+1}-a_{k}}{2}\right)^{\alpha / 2} 2 \sqrt{c}\right)^{1 / M-1}\right) \leq \infty
$$

be satisfied. If for some $\widehat{\varepsilon}, \widehat{\varepsilon} \geq \varepsilon^{*}$, the following series

$$
\sum_{k=0}^{\infty} \exp \left\{-\frac{\left(c\left(a_{k}\right)-c\left(a_{0}\right)\right) \widehat{\varepsilon}}{\sqrt{2} \sigma^{2}}\right\}\left(1+\frac{\sqrt{2} c\left(a_{k}\right) \widehat{\varepsilon}}{\sigma^{2}}\right)^{1 / 2}<\infty
$$

then for all $\varepsilon>\widehat{\varepsilon}$

$$
P\left\{\sup _{0 \leq t \leq \infty} \frac{\left.|| X(t)\right|^{2}-\sigma^{2} \mid}{c(t)}>\varepsilon\right\} \leq \exp \left\{-\frac{\sqrt{2} c\left(a_{0}\right) \varepsilon}{\sigma^{2}}\right\} \cdot \widehat{z}=Z(\varepsilon)
$$

where

$$
\widehat{z}=4 e^{\frac{M+1}{\alpha}} \cdot\left(\frac{2}{M}\right)^{\frac{2 M}{\alpha}} \sum_{k=0}^{\infty} \exp \left\{-\frac{\left(c\left(a_{k}\right)-c\left(a_{0}\right)\right) \widehat{\varepsilon}}{\sqrt{2} \sigma^{2}}\right\}\left(1+\frac{\sqrt{2} c\left(a_{k}\right) \widehat{\varepsilon}}{\sigma^{2}}\right)^{1 / 2} .
$$

Proof

For $Y(t)=|X(t)|^{2}-\sigma^{2}$ we have that $\operatorname{Var}(Y(t)-Y(s)) \leq 2 \sigma^{2}|t-s|^{\frac{\alpha}{2}}$ (see proof of Theorem 5.2). It follows 
from Theorem 5.2 that for $M>1$ and

$$
u>\frac{2 \sqrt{2} \sigma^{2} M}{\alpha}\left(\max \left(1,\left(\left(\frac{a_{k+1}-a_{k}}{2}\right)^{\frac{\alpha}{2}} 2 \sqrt{c}\right)^{\frac{1}{M-1}}\right)\right)
$$

we have

$$
P\left\{\sup _{a_{k} \leq t \leq a_{k+1}}|Y(t)|>u\right\} \leq 4 e^{\frac{M+1}{\alpha}} \exp \left\{-\frac{u}{\sqrt{2} \sigma^{2}}\right\}\left(\frac{\alpha u}{2 \sqrt{2} \sigma^{2} M}\right)^{\frac{2 M}{\alpha}}\left(1+\frac{\sqrt{2} u}{\sigma^{2}}\right)^{1 / 2}
$$

The following inequality is obvious.

$$
P\left\{\sup _{t \in[0, \infty)} \frac{|Y(t)|}{c(t)}>\varepsilon\right\} \leq \sum_{k=0}^{\infty} P\left\{\sup _{a_{k} \leq t \leq a_{k+1}}|Y(t)|>c\left(a_{k}\right) \cdot \varepsilon\right\} .
$$

It follows from (19) and (20) that for

$$
\varepsilon>\frac{2 \sigma M}{\alpha c\left(a_{k}\right)} \cdot\left(\max \left(1,\left(\left(\frac{a_{k+1}-a_{k}}{2}\right)^{\frac{\alpha}{2}} 2 \sqrt{c}\right)^{\frac{1}{M-1}}\right)\right),
$$

we have the following estimate

$$
P\left\{\sup _{a_{k} \leq t \leq a_{k+1}}|Y(t)|>c\left(a_{k}\right) \varepsilon\right\} \leq 4 e^{\frac{M+1}{\alpha}} \exp \left\{-\frac{c\left(a_{k}\right) \varepsilon}{\sqrt{2} \sigma^{2}}\right\}\left(\frac{\alpha c\left(a_{k}\right) \varepsilon}{2 \sqrt{2} \sigma^{2} M}\right)^{\frac{2 M}{\alpha}}\left(1+\frac{\sqrt{2} c\left(a_{k}\right) \varepsilon}{\sigma^{2}}\right)^{1 / 2} .
$$

From this inequality (23) and inequality (21) it follows that under condition (22) we have the following estimate

$$
\begin{aligned}
& P\left\{\sup _{a_{k} \leq t \leq a_{k+1}} \frac{|Y(t)|}{c(t)}>\varepsilon\right\} \leq 4 e^{\frac{M+1}{\alpha}} \sum_{k=0}^{\infty} \exp \left\{-\frac{c\left(a_{k}\right) \varepsilon}{\sqrt{2} \sigma^{2}}\right\}\left(\frac{\alpha c\left(a_{k}\right) \varepsilon}{2 \sqrt{2} \sigma^{2} M}\right)^{\frac{2 M}{\alpha}}\left(1+\frac{\sqrt{2} c\left(a_{k}\right) \varepsilon}{\sigma^{2}}\right)^{1 / 2}= \\
& =4 e^{\frac{M+1}{\alpha}} \exp \left\{-\frac{c\left(a_{0}\right) \varepsilon}{\sqrt{2} \sigma^{2}}\right\} \sum_{k=0}^{\infty} \exp \left\{-\frac{\left(c\left(a_{k}\right)-c\left(a_{0}\right)\right) \varepsilon}{\sqrt{2} \sigma^{2}}\right\}\left(\frac{\alpha c\left(a_{k}\right) \varepsilon}{2 \sqrt{2} \sigma^{2} M}\right)^{\frac{2 M}{\alpha}}\left(1+\frac{\sqrt{2} c\left(a_{k}\right) \varepsilon}{\sigma^{2}}\right)^{1 / 2}
\end{aligned}
$$

It follows from (22) that $\varepsilon>\frac{2 \sigma M}{\alpha c\left(a_{k}\right)}$ and $\frac{\alpha c\left(a_{k}\right)}{2 \sigma} \varepsilon>M \geq 2$.

From inequality (24) under condition (22) we have the following estimate

$$
\begin{gathered}
P\left\{\sup _{t \in[0, \infty)} \frac{|Y(t)|}{c(t)}>\varepsilon\right\} \leq 4 e^{\frac{M+1}{\alpha}}\left(\frac{2}{M}\right)^{\frac{2 M}{\alpha}} \exp \left\{-\frac{c\left(a_{0}\right) \varepsilon}{\sqrt{2} \sigma^{2}}\right\} \times \\
\times \sum_{k=0}^{\infty} \exp \left\{-\frac{\left(c\left(a_{k}\right)-c\left(a_{0}\right)\right) \varepsilon}{\sqrt{2} \sigma^{2}}\right\}\left(1+\frac{\sqrt{2} c\left(a_{k}\right) \varepsilon}{\sigma^{2}}\right)^{1 / 2} .
\end{gathered}
$$

The function $f(\varepsilon)=\exp \left\{-\frac{\left(c\left(a_{k}\right)-c\left(a_{0}\right)\right) \varepsilon}{\sigma}\right\}\left(1+\frac{c\left(a_{k}\right) \varepsilon}{\sigma}\right)^{1 / 2}$ monotonically decreases for $\varepsilon>0$. For this reason under the condition

$$
\hat{\varepsilon} \geq \varepsilon^{*}
$$

we have that $\forall \varepsilon>\widehat{\varepsilon}$

$$
\begin{gathered}
P\left\{\sup _{t \in[0, \infty)} \frac{|Y(t)|}{c(t)}>\varepsilon\right\} \leq 4 e^{\frac{M+1}{\alpha}}\left(\frac{2}{M}\right)^{\frac{2 M}{\alpha}} \times \\
\times \exp \left\{-\frac{c\left(a_{0}\right) \varepsilon}{\sqrt{2} \sigma^{2}}\right\} \sum_{k=0}^{\infty} \exp \left\{-\frac{\left(c\left(a_{k}\right)-c\left(a_{0}\right)\right) \hat{\varepsilon}}{\sqrt{2} \sigma^{2}}\right\}\left(1+\frac{\sqrt{2} c\left(a_{k}\right) \hat{\varepsilon}}{\sigma^{2}}\right)^{1 / 2} .
\end{gathered}
$$




\section{Corollary 6.2}

Let a function $c(t)$ satisfies conditions of Theorem 6.2. Then with probability one for all $t \in \mathbb{R}$ the following inequality is satisfied

$$
|Y(t)| \leq \eta \cdot c(t)
$$

where $\eta>0$ is a random variable such that for $\varepsilon>\widehat{\varepsilon}$ the inequality

$$
P\{\eta>\varepsilon\} \leq Z(\varepsilon)
$$

holds true. For definition of the function $Z(\varepsilon)$ see (18).

Remark 6.2. Condition (17) is satisfied if the series

$$
\sum_{k=0}^{\infty} \exp \left\{-\frac{c\left(a_{k}\right) \hat{\varepsilon}}{\sqrt{2} \sigma^{2}}\right\}\left(c\left(a_{k}\right)\right)^{\frac{2 M}{\alpha}+\frac{1}{2}}
$$

converges. This series converges, for example, in the case where $c\left(a_{k}\right)=\ln \left(k^{d}\right), k>1$, where $\frac{d \widehat{\varepsilon}}{\sqrt{2} \sigma^{2}}>1$. A special case is $a_{k}=k$, that is $c(t)=d \ln (t), d>\frac{\sqrt{2} \sigma^{2}}{\widetilde{\varepsilon}}$ and $t>e$.

\section{Conclusions}

In the article analysis of properties of proper complex random process is presented. Definitions and some properties of proper stationary random complex process with stable covariance function are given. Estimates of distribution of some functionals from module of stationary Gaussian proper complex random processes are obtained. Behaviour of the module of stationary proper complex random processes at infinity is analysed.

\section{Acknowledgements}

The authors would like to thank professor Mikhail Moklyachuk for valuable discussions which helped to substantially improve the quality of the paper and an anonymous referee for the constructive propositions.

\section{REFERENCES}

1. S. A. Akhmanov, J. E. Deacons, A. S. Chirkin, Introduction to Statistical Radiophysics and Optics, M.: Science, (1981). (in Russian)

2. J. L. Doob, Stochastic Processes, John Wiley \& Sons, (1953).

3. Yu. Kozachenko, V.V.Buldygin, Metric Characterization of Random Variables and Random processes, American Mathematical Society, Providence, Rhode Island, (2000).

4. Yu. Kozachenko, Random processes in Orlicz spaces. I, Theor. Probability and Math. Statist., vol. 30, pp. 92-107, 1984. (in Ukrainian)

5. Yu. Kozachenko, M.M.Perestyuk O.I.Vasylyk On uniform convergence of wavelet expansions of -sub-Gaussian random processes, Random Oper. And Stoch. Equations, vol. 14, no. 3, pp. 209-232, 2006.

6. Yu. Kozachenko, I.Rozora, Simulation of Gaussian stochastic processes, Random Oper. And Stoch. Equations, vol. 11, no. 3, pp. 275-296, 2003.

7. Yu. Kozachenko, O. Pogorilyak, I. Rozora, A. Tegza, Simulation of Stochastic Processes with Given Accuracy and Reliability, ISTE Press - Elsevier, 2016.

8. Yu. Kozachenko, O.V.Stus, Square-Gaussian Random Processes and Estimators of Covariance Functions, Math. Communications, vol. 3, no. 1, pp. 83-94, 1998.

9. Yu. Kozachenko, V.Troshki, A criterion for testing hypotheses about the covariance function of a stationary Gaussian stochastic proses, Modern Stochastics Theory and Application, vol. 1, no. 2, pp. 139-149, 2014.

10. Yu. Kozachenko, O. Vasylyk R. Jamnenko, Upper estimate of overrunning by Sub $(\Omega)$ random process the level specified by continuous function, Math. Communications, vol. 3, no. 1, pp. 83-94, 1998.

11. E. Lukacs, Characteristic Functions, New York: Hafner Pub. Co., 1970.

12. F. D. Neeser, and J. L. Massey, Proper complex random processes with applications to information theory, IEEE Transactions on Information Theory, vol. 39, issue: 4, pp. 1293 - 1302, 1993.

13. M. Petranova, Simulation of Gaussian stationary quasi Ornstein-Uhlenbeck process with given reliability and accuracy in spaces $C([0, T])$ and $L p([0, T])$, Journal of Applied Mathematics and Statistics. - vol. 3, no. 1, pp. 144-58, 2016.

14. P. A. Vatnik, Statistical methods of operational management of prodaction, M .: Statistics, (1978). (in Russian) 